\title{
Sentinel node procedure under local anesthesia in breast cancer patients. Lessons learned from a prematurely closed randomized clinical trial
}

\author{
Martijn Leenders ${ }^{1 *}$, Shirley Go ${ }^{2}$, Alexander Lopes Cardozo ${ }^{1}$, Robbert Mollema ${ }^{1}$ and Hermien Schreurs ${ }^{1}$
}

*Correspondence: martijn_leenders@hotmail.com

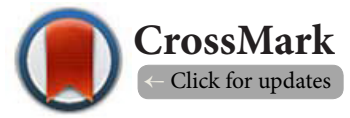

'Department of Surgery, Northwest Clinics, Wilhelminalaan 12, 1815 JD Alkmaar, The Netherlands.

${ }^{2}$ Department of Radiology, Northwest Clinics, Wilhelminalaan 12, 1815 JD Alkmaar, The Netherlands.

\begin{abstract}
Purpose: Ideally, sentinel node status is known prior to breast surgery in breast cancer patients, so a treatment plan can be made before breast surgery. If indicated, a one-step surgical procedure can then be performed (breast surgery and axillary lymph node dissection at once). Previously, we demonstrated that $36.5 \%$ of breast cancer patients younger than 60 years with a breast carcinoma larger than 20 millimeters have macrometastatic axillary nodes. Therefore, we aimed that sentinel node procedure (SNP) under local anesthesia (LA) reduces the number of two-stage surgical procedures in these patients with 30\%.

Methods: The AMBULANT study was a prospective trial examining patients aged 18 to 60 years with a breast carcinoma larger than 20 millimeters and without clinically or ultrasound-guided cytological evidence of axillary node involvement. Patients were randomized to undergo SNP under LA versus usual care. Number of operations, periprocedural parameters and psychological outcomes were evaluated.

Results: The trial was prematurely closed, because of lower than expected patient accrual and new insights on breast cancer treatment. Twelve patients were included; five patients were randomized to SNP under LA and seven to usual care. Baseline characteristics were similar. SNP under LA did not reduce the number of twostage surgical procedures. Duration of SNP was significantly longer when performed under LA and three of five patients experienced too much pain during SNP under LA. Finally, SNP under LA negatively influences physical health.
\end{abstract}

Conclusion: SNP under LA does not reduce the number of two-stage surgical procedures in breast cancer patients.

Keywords: Local Anesthesia, Breast Neoplasms, Randomized Controlled Trial, Sentinel Lymph Node

\section{Introduction}

In breast cancer patients, lymph node status is a key prognostic indicator [1]. Axillary ultrasound (US) with subsequent fine needle aspiration cytology (FNAC) of suspicious lymph nodes (US/FNAC) is widely used for axillary staging purposes. The operative technique of choice to stage the axilla is the sentinel node procedure (SNP). When either US/FNAC or SNP reveals lymph node metastases, an axillary lymph node dissection (ALND) has long been regarded as the standard treatment. However, emerging evidence shows that ALND does not affect disease-free survival and overall survival in early breast cancer patients with limited disease in their sentinel node(s) [2-4]. Consequently, to decide if axillary treatment (ALND or axillary radiotherapy) is recommended in node-positive breast cancer patients, axillary tumor load should be known. This axillary tumor load can only be reliably evaluated by SNP, not by US/ FNAC. Therefore, the role of SNP might become increasingly important in upcoming years. Ideally, the status of the sentinel lymph node is known prior to breast surgery, so a patient tailored (axillary) treatment plan can be made and discussed with the patient before breast surgery. Moreover, if an ALND should be performed as a consequence of the SNP, a one-step 
surgical procedure can be performed (breast surgery and ALND during the same procedure). In this context, SNP under local anesthesia (LA), prior to breast surgery, seems very attractive. The effectiveness and liability of preoperative SNP under LA have been studied by various study groups. The general conclusion is that SNP under LA is a safe procedure that does not harm long-term locoregional control [5-9]. In the vast majority of breast cancer patients, SNP under LA will not lead to treatment changes, because most patients are sentinel node negative. Hence, performing SNP under LA in all breast cancer patients is not useful. Previously, we analyzed which subgroup of breast cancer patients could potentially benefit from SNP under LA. We concluded that approximately $36.5 \%$ of breast cancer patients younger than 60 years with a primary breast carcinoma larger than 20 millimeters and with a negative US/FNAC result did have one or more macrometastatic sentinel nodes histologically [10]. These breast cancer patients with poor prognostic features are usually treated with either completion ALND or axillary radiotherapy. Hence, those are the patients that could benefit from a SNP under LA. Additionally, young patients are in particular risk for depressive symptoms the first three months after their cancer diagnosis [11-13]. Knowing lymph node status and the complete treatment plan in an early phase of breast cancer diagnosis might be beneficial with regards to psychological distress in these young breast cancer patients. Therefore, we compared SNP under LA with usual care (SNP during breast cancer surgery) in breast cancer patients aged 18 to 60 years with a breast cancer larger than 20 millimeters and with a negative US/FNAC. The primary aim of this study was to reduce the number of two-stage surgical procedures under general anesthesia with $30 \%$.

\section{Methods}

\section{Patient characteristics}

This single-center, randomized phase 3 trial was registered with ClinicalTrials.gov (number NCT02187718) and approved by the local Medical Ethical Review Board (METC Noord-Holland) and by the Investigational Review Board of the Northwest Clinics. All patients provided written informed consent. Women aged 18 to 60 with histologically confirmed invasive breast carcinoma sonographically larger than 20 millimeters, preoperative negative US/FNAC, no evidence of distant metastases and ASA Classification I to III were eligible for participation. Patients who previously had received treatment of the breast or axilla by surgery or radiotherapy on the affected side, patients who were to be treated by neoadjuvant therapy and patients who were allergic to lidocaine were ineligible.

\section{Study design and treatment}

Eligible women were randomly (1:1) assigned to SNP under LA or usual care. Preoperative work-up was identical for both groups. Patients entered the study following a negative axillary US/FNAC. In the experimental group, SNP under LA was performed within one week after randomization. Two to 24 hours prior to the SNP under LA, a radioactive isotope (Tc99m colloidal albumin) was injected in the periareolar region of the affected breast. Lymphoscintigraphy was performed to inform on the number and location of focal accumulations of radiocolloid in lymph nodes. The SNP under LA was performed in our outpatient operating rooms. No premedication was administered. Venous access was provided for all patients. Patent Blue $V^{\circledR}$ dye (Guerbet, Aulnay-Sous Bois, France) was injected in the periareolar region of the diseased breast. Next, the location of the sentinel node was marked on the skin using the gamma probe (Neoprobe 2000). Local anesthesia was administered by subcutaneous infiltration of lidocaine $(0.5 \%)$ with adrenaline (1:200.000). If needed, more anesthetic was injected during the procedure. The sentinel node(s) was identified and harvested guided by the lymphoscintigraphy, the blue lymphatic vessels and detection of radioactivity by the gamma probe. Within one week after the SNP under LA, histological results of the SNP were discussed in our multidisciplinary meeting (MDT) and the need for ALND was discussed. The decision whether to perform an ALND was not influenced by participation in this study. Resection of the breast carcinoma with possible ALND was planned within three weeks after randomization. Patients in the control group were planned for resection of their breast cancer combined with SNP under general anesthesia within three weeks after randomization as well. The SNP in this group is identical to the procedure described above with the only exception that no local anesthetic is injected. If an ALND was indicated in the control group, this operation was planned within six weeks after randomization. Pathological examination, the operation method used to excise the breast cancer and the administration of adjuvant systemic therapy or radiotherapy were not influenced by participation in this study.

\section{Study end points}

The primary end point was the number of operations under general and under local anesthesia per patient. Secondary end points were quality of life (QoL), depressive symptoms and anxiety experienced by patients. Additionally, periprocedural parameters were collected. The amount of pain during SNP under LA was measured with a Numeric Pain Rating Scale (NPRS). A score of 4 or higher on the 11-item NPRS was defined as too much pain. The total amount of lidocaine used during SNP under LA was noted as well. At last, the number of excised lymph nodes, histopathology of excised lymph nodes, duration of SNP and surgical morbidity were compared between both groups.

\section{Questionnaires}

Questionnaires were completed at baseline $(t=0)$, within 48 hours after the breast operation ( $t=21)$ and three $(t=90)$ and six months ( $\mathrm{t}=180$ ) after randomization. Personality (STAl-trait) was assessed at baseline. Experienced momentary anxiety 
Leenders et al., Breast Cancer Reports 2020,

(STAI-state), depressive symptoms (CES-D) and quality of life (WHOQOL-bref) were assessed at $\mathrm{t}=0, \mathrm{t}=21, \mathrm{t}=90$ and $\mathrm{t}=180$. The "State-and-Trait-Anxiety-Inventory" (STAI) measures state and trait anxiety. The personality characteristic trait anxiety concerns differences in individuals in the disposition to respond to stressful situations with varying amounts of stress. State anxiety refers to the amount of stress being experienced at the specific moment the measurement is made. In this study, the 10-item short version of the STAI-Trait scale and the 6-item short version of the STAI-State scale were used. Scores of both tests range from 20 to 80 . The mean score is 34 to 36 for working adults [14]. The reliability and validity of the short versions are considered good [15]. QoL was assessed using the 26-item version of the WHO quality of life questionnaire (WHOQOL-bref). The WHOQOL-bref measures QoL in four domains (physical health, psychological health, social relationships and environment) and the facet overall QoL and general health. The four domain scores range from 0 to 100 . Psychometric properties of the WHOQOL-bref are good [16]. Depressive symptoms were assessed using the CESD questionnaire developed by the "Center for Epidemiologic Studies". This questionnaire focuses on the situation in the past week. It does not assess chronic depression but only depressive symptoms that could have developed as the result of a certain event. Scores range from 0 to 60 . A score of 16 or higher is considered as an indicator of clinical depression. Psychometric properties are good [17-19].

\section{Statistical analysis}

The target accrual for the trial was 52 patients to confirm $30 \%$ reduction of two-stage surgical procedures with $80 \%$ power (type II error 0.2) and a 5\% significance level (type I error of 0.05). In a two-stage surgical procedure excision of the breast carcinoma and a possible ALND are performed in two separate operations. In a one-stage surgical procedure the breast operation and ALND are performed simultaneously. To describe measurements, median and IQR (Inter Quartile Range), were used. For examining differences between the two groups, chi-square and Mann Whitney $\mathrm{U}$ tests were used. A linear mixed effects model was used to examine scores on QoL, state anxiety and depressive symptoms over time. A pvalue of 0.05 or less was considered statistically significant. All analyses were performed using SPSS version 25.0 (Statistical Package for Social Sciences ${ }^{\circledast}$ ).

\section{Results}

\section{Patient characteristics}

The first patient was enrolled in January 2015. The trial was prematurely closed in November 2015 because of lower than expected patient accrual and new insights on breast cancer treatment. Between January and November 2015, all breast cancer patients treated in the Northwest Clinics (location Alkmaar) were screened for eligibility for participation in this trial. Forty-four patients were eligible. Because of varying reasons, 32 patients were excluded, leaving 12 patients for randomization (Figure 1). Five patients were randomly assigned to the SNP under LA group and 7 patients to the usual care group (Figure 1). Patient and disease characteristics were well balanced between the two groups (Table 1).

Table 1. Baseline characteristics by study group.

\begin{tabular}{lll}
\hline & \multicolumn{2}{c}{ No. (\%) } \\
\hline Characteristic & SNP under LA & Usual Care \\
\hline Age & $48(14)$ & $49(13)$ \\
Median (IQR), years & & \\
\hline Clinical tumor size & $23.0(180)$ & $21.0(3.0)$ \\
Median (IQR), mm & $5(100 \%)$ & $7(100 \%)$ \\
cT2 & & \\
\hline Type & $3(60 \%)$ & $6(86 \%)$ \\
Infiltrating ductal & $2(40 \%)$ & $1(14 \%)$ \\
Infiltrating lobular & & \\
Grade (Bloom and Richardson) & $2(40 \%)$ & $2(29 \%)$ \\
II & $3(50 \%)$ & $5(71 \%)$ \\
III & & \\
\hline Receptor Status & $1(20 \%)$ & $1(14 \%)$ \\
ER+/PR+/Her2+ & $2(40 \%)$ & $5(71 \%)$ \\
ER+/PR+/Her2- & $1(20 \%)$ & $0(0 \%)$ \\
ER+/PR-/Her2- & $1(20 \%)$ & $1(14 \%)$ \\
ER-/PR-/Her2- & & \\
\hline Type of Breast Surgery & $4(80 \%)$ & $6(86 \%)$ \\
Breast-conserving surgery & $1(20 \%)$ & $1(14 \%)$ \\
Mastectomy & $32.0(8.0)$ & $32.0(12.0)$ \\
\hline Psychological factors median scores $(I Q R)$ & $63.3(16.7)$ \\
\hline Trait-anxiety at baseline & $36.7(18.3)$ & $17.0(15.0)$ \\
State-anxiety at baseline & $11.0(12.5)$ & \\
QoL (Physical Health) at baseline & \\
Depressive symptoms at baseline & & \\
\hline & & \\
\hline
\end{tabular}

No.=number; $\mathrm{SNP}=$ sentinel node procedure; $\mathrm{LA}=$ local anesthesia; $\mathrm{IQR}=$ Inter Quartile Range; ER=estrogen receptor; $\mathrm{PR}=$ progesterone receptor; Her2=human epidermal growth factor receptor 2; QoL=quality of life.

\section{Sentinel node procedure}

In the SNP under LA group, a median number of 1 sentinel node (IQR 2) was harvested. A similar number of sentinel nodes was harvested in the usual care group (median 1, IQR 1) $(p=0.760)$. The SNP under LA took significantly longer than the SNP under general anesthesia, that is a median of 25 minutes (IQR 7.5 minutes) versus 20 minutes (IQR 4 minutes) $(p=0.010)$. On average, 15 milliliters of lidocaine $0.5 \%$ was used during the SNP under LA. In the SNP under LA group, three of five patients had a score of four or higher on the NPRS and thus experienced too much pain during SNP under LA. There were no complications related to the SNP in either one of the groups (Table 2).

\section{Number of operations}

In the SNP under LA group, histopathologic examination of the sentinel nodes showed no metastases in 4 out of 5 patients $(80 \% \mathrm{pN} 0)$ and a micrometastasis in the sentinel node of one 


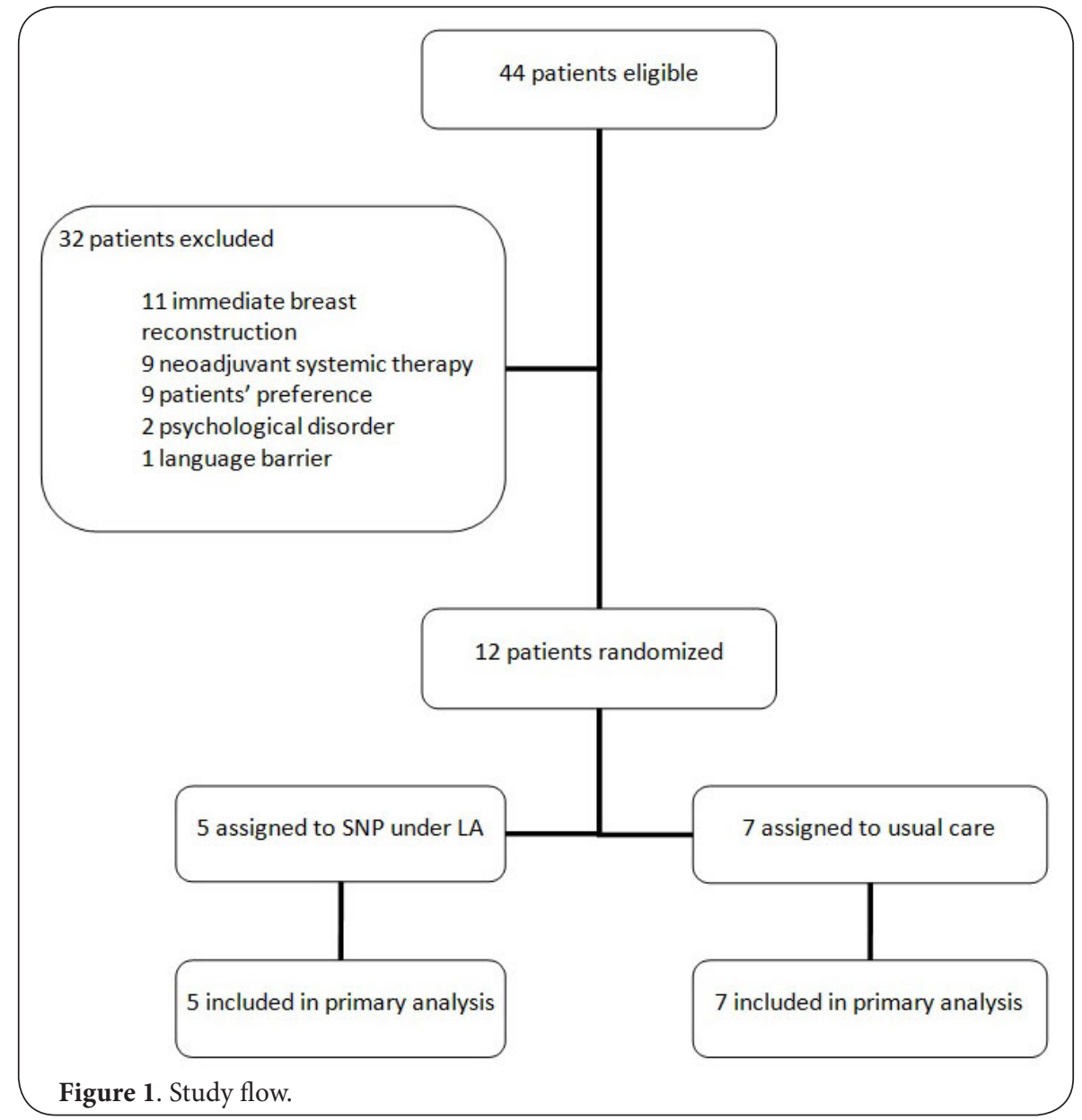

Table 2. Results of sentinel node procedures in both groups.

\begin{tabular}{llll}
\hline & SNP under LA & Usual Care & $\mathbf{p}$ \\
\hline Sentinel Node Procedure & & & \\
No. of SNPs & 5 & 7 & \\
Total no. of sentinel nodes & 9 & 11 & \\
No. of sentinel nodes, median (IQR) & $1(2)$ & $1(1)$ & 0.760 \\
Duration of SNP, median (IQR), min & $25(7.5)$ & $20(4)$ & $\mathbf{0 . 0 1 0}$ \\
Lidocaine, median (IQR), mL & $15(5)$ & -- & \\
NPRS, median (IQR) & $4(2.5)$ & -- & \\
\hline Histology of sentinel nodes & & & \\
No metastasis & $4(80 \%)$ & $5(71 \%)$ & \\
Micrometastasis & $1(20 \%)$ & $0(0 \%)$ & \\
Macrometastasis & $0(0 \%)$ & $2(29 \%)$ & \\
\hline
\end{tabular}

$\mathrm{SNP}=$ sentinel node procedure; $\mathrm{LA}=$ local anesthesia; No.=number; $\mathrm{IQR}=$ Inter Quartile Range; $\mathrm{mL}=$ milliliters; NPRS=11-item Numeric Pain Rating Scale

patient ( $20 \%$ pN1 mi). The patient with the micrometastasis in her sentinel node was treated with regional node irradiation (axillary levels I/II) and adjuvant systemic therapy. No ALND was performed. In the usual care group, histopathological examination of the sentinel nodes showed no metastases in 5 out of 7 patients ( $71 \%$ pN0) and macrometastases in the sentinel nodes of two patients ( $29 \%$ pN1a) (Table 2$)$. The patients with macrometastases in their sentinel nodes were both treated with regional node irradiation (axillary levels I/ II) and adjuvant systemic therapy. No ALND was performed in these patients. Accordingly, the number of operations under general anesthesia per patient was one in both groups. The number of operations under local anesthesia per patient was one in the SNP under LA group and zero in the usual care group. Since there were no two-stage surgical procedures in the usual care group, the number of two-stage surgical 
Leenders et al., Breast Cancer Reports 2020,

http://www.hoajonline.com/journals/pdf/2057-1631-7-3.pdf

doi: $10.7243 / 2057-1631-7-3$

procedures under general anesthesia is not reduced by performing the SNP under local anesthesia in this trial.

\section{Quality of Life, anxiety and depressive symptoms}

Mean scores of the questionnaires during follow-up were analyzed for all patients collectively (regardless of the group in which they were randomized) as well as separately for both groups. The baseline scores of the QoL domains were comparable between both groups. When analyzing all patients collectively, the physical health domain scores of $\mathrm{QoL}$ significantly diminished at $\mathrm{t}=21(p=0.015)$ and $\mathrm{t}=180$ $(p=0.006)$ compared with baseline scores (Table 3A). When analyzing the SNP under LA and usual care group separately, these lower physical health domain scores during follow-up were only noted in the SNP under LA group, not in the usual care group (Table 3B). Mean physical health domain scores are graphically depicted in Figure 2 . With regards to anxiety scores, both baseline trait anxiety and baseline state anxiety scores did not differ between the two groups. When analyzing all patients collectively, state anxiety scores were significantly lower at $\mathrm{t}=21(p=0.05)$, three months $(p=0.012)$ and six months $(p=0.031)$ compared with baseline scores. When analyzing the SNP under LA and usual care group separately, only the state anxiety score at $\mathrm{t}=21$ was significantly lower compared with baseline in the usual care group $(p=0.026$, Table 3B). Concerning depressive symptoms, there were no significant differences between both groups at any time nor did depressive symptoms change during follow-up in both groups (Table 3).

\section{Discussion}

At the time of initiation of this randomized clinical trial, young breast cancer patients with primary breast carcinomas larger than two centimeters and one or more macrometastatic lymph nodes were usually treated with either completion axillary lymph node dissection or axillary radiotherapy. Based on this common practice and the results of a retrospective analysis of our group (that is $36.5 \%$ of breast cancer patients younger than 60 years with a breast carcinoma larger than 20 millimeters and with a negative US/FNAC did have histologically one or more macrometastatic sentinel nodes [10]), we hypothesized that we could reduce the number of two-stage surgical procedures under general anesthesia with $30 \%$ by performing a SNP under local anesthesia in this subgroup of breast cancer patients.

While preparing and actually starting this study, new insights on treatment of breast cancer patients with sentinel node metastases rapidly evolved. First, the results of the ACOSOG Z0011, IBCSG 23-01 and AATRM 048/13/2000 studies [2-4] comparing ALND versus no ALND in sentinel node positive breast cancer patients were rapidly implemented in daily practice. Although only the ACOSOG Z0011 trial included patients with sentinel node macrometastases [2] and the quality of evidence is moderate, less and less breast cancer

Table 3. Scores on Quality of Life, State anxiety and Depressive Symptoms.

A. All patients.

\begin{tabular}{|c|c|c|c|c|}
\hline \multirow{3}{*}{ Mean scores (SD) } & \multicolumn{4}{|l|}{ All patients } \\
\hline & $t=0$ & $t=21$ & $t=90$ & $t=180$ \\
\hline & $\mathrm{n}=12$ & $\mathrm{n}=12$ & $\mathrm{n}=10$ & $\mathrm{n}=9$ \\
\hline QoL - Physical domain & $76.7(12.1)$ & $63.7(18.2)^{\mathrm{a}}$ & $68.9(19.1)$ & $61.2(17.1)^{\mathrm{b}}$ \\
\hline State Anxiety & $45.0(9.1)$ & $36.9(8.1)^{c}$ & $38.0(9.8)^{\mathrm{d}}$ & $38.1(7.5)^{\mathrm{e}}$ \\
\hline Depressive Symptoms & $13.6(8.1)$ & $13.8(8.0)$ & $11.4(7.0)$ & $12.2(9.1)$ \\
\hline \multicolumn{5}{|c|}{$\begin{array}{l}S D=\text { standard deviation; } n=\text { number of patients; } Q o L=\text { Quality of Life } \\
{ }^{\text {a }} \text { score significantly changed at } \mathrm{t}=21 \text { compared with } \mathrm{t}=0(p=0.015) \\
{ }^{\mathrm{b}} \text { score significantly changed at } \mathrm{t}=180 \text { compared with } \mathrm{t}=0(p=0.006) \\
{ }^{\mathrm{c}} \text { score significantly changed at } \mathrm{t}=21 \text { compared with } \mathrm{t}=0(p=0.05) \\
{ }^{\mathrm{d}} \text { score significantly changed at } \mathrm{t}=90 \text { compared with } \mathrm{t}=0(p=0.012) \\
{ }^{\mathrm{e}} \text { score significantly changed at } \mathrm{t}=180 \text { compared with } \mathrm{t}=0(p=0.031)\end{array}$} \\
\hline
\end{tabular}

B. Comparing SNP under LA group and usual care group.

\begin{tabular}{lllllllll}
\hline \multicolumn{7}{l}{ SNP under LA } & \multicolumn{7}{l}{ Usual Care } \\
\hline Mean scores (SD) & $\mathrm{t}=0$ & $\mathrm{t}=21$ & $\mathrm{t}=90$ & $\mathrm{t}=180$ & $\mathrm{t}=0$ & $\mathrm{t}=21$ & $\mathrm{t}=90$ & $\mathrm{t}=180$ \\
& $\mathrm{n}=5$ & $\mathrm{n}=5$ & $\mathrm{n}=4$ & $\mathrm{n}=5$ & $\mathrm{n}=7$ & $\mathrm{n}=7$ & $\mathrm{n}=6$ & $\mathrm{n}=4$ \\
\hline QoL - Physical Domain & $83.6(9.5)$ & $64.0(23.0)^{\mathrm{a}}$ & $65.8(22.0)$ & $56.4(14.6)^{\mathrm{b}}$ & $71.7(11.7)$ & $63.4(16.0)$ & $71.0(18.8)$ & $67.3(20.3)$ \\
\hline State Anxiety & $42.7(10.9)$ & $33.3(11.3)$ & $32.5(10.0)$ & $36.7(5.8)$ & $46.7(7.9)$ & $39.5(4.1)^{\mathrm{c}}$ & $41.7(8.6)$ & $40.0(9.8)$ \\
\hline Depressive Symptoms & $10.4(6.8)$ & $9.2(6.7)$ & $11.3(7.1)$ & $13.0(9.0)$ & $15.9(8.6)$ & $17.0(7.5)$ & $11.5(7.6)$ & $11.3(10.6)$ \\
\hline
\end{tabular}

$S N P=$ sentinel node procedure; $L A=$ local anaesthesia; $S D=$ standard deviation; $n=$ number of patients; QoL = Quality of Life

${ }^{a}$ score significantly changed at $\mathrm{t}=21$ compared with $\mathrm{t}=0(p=0.023)$

${ }^{\mathrm{b}}$ score significantly changed at $\mathrm{t}=180$ compared with $\mathrm{t}=0(p=0.004)$

${ }^{c}$ score significantly changed at $\mathrm{t}=21$ compared with $\mathrm{t}=0 \quad(p=0.026)$ 


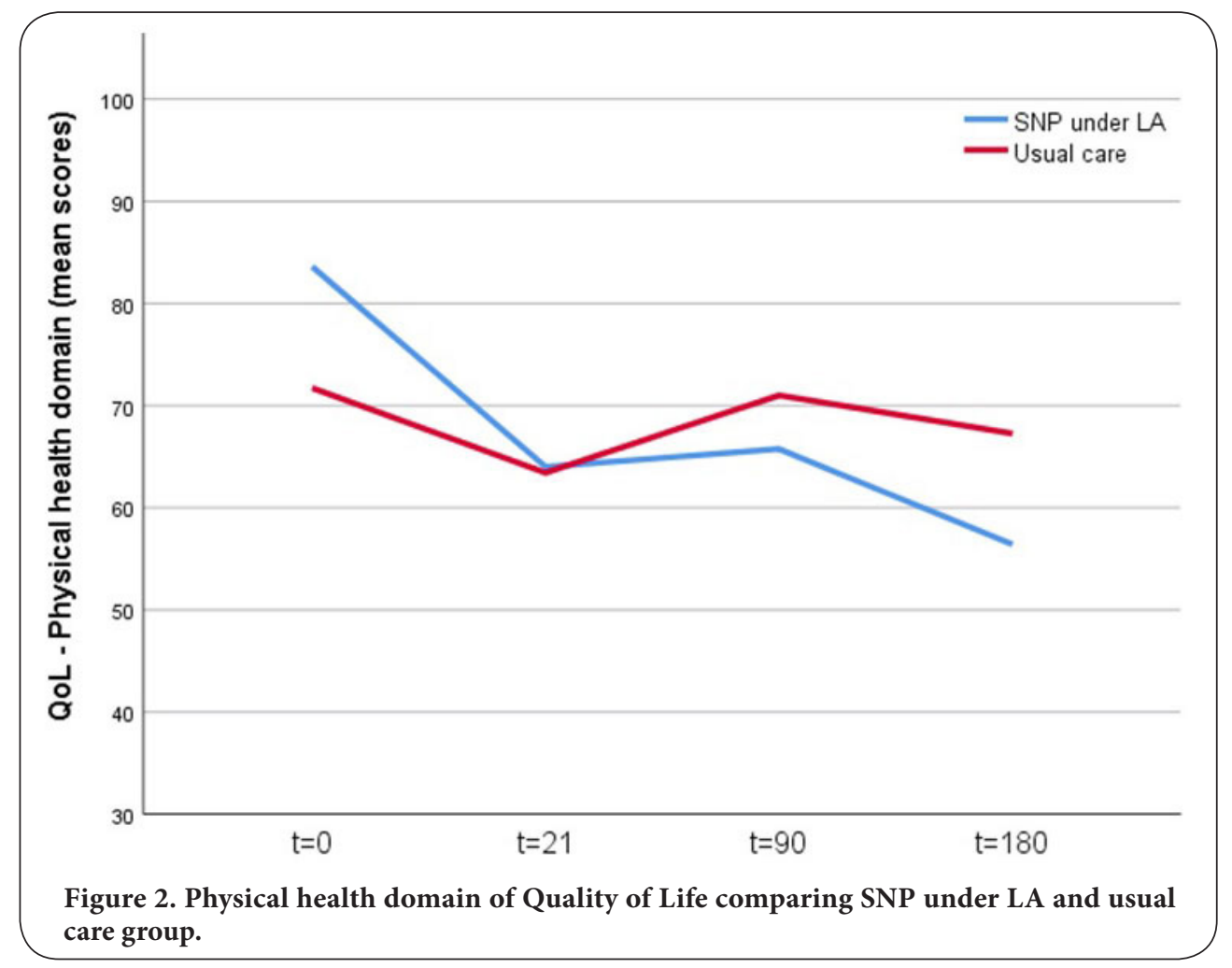

patients with macrometastatic sentinel nodes were treated with an ALND. Consequently, most patients who met our inclusion criteria and did have one or two macrometastatic sentinel nodes, were not to be treated with an ALND. Second, breast cancer patients receive neoadjuvant systemic therapy with increasing frequency. Particularly in the subset of breast cancer patients that we were interested in. Partly because of this increased use of neoadjuvant systemic therapy, patient accrual was lower than expected. Another reason for the slow patient accrual were the physicians' preferences to perform SNP under LA in patients in whom mastectomy with immediate breast reconstruction was planned. By knowing sentinel node status preoperatively, the chance of post-mastectomy radiotherapy can better be estimated. In our hospital, immediate breast reconstruction is preferably not performed when post-mastectomy radiotherapy is administered, because of the increased chance of complications [20].

These new insights on breast cancer treatment combined with slow patient accrual decided us to close this randomized controlled trial after inclusion of only twelve patients. In this article we analyzed the results of these twelve patients. Considering the explanation above, it is quite logical that the percentage of two-stage surgical procedures under general anesthesia was not reduced at all by performing SNP under LA, though it did result in one additional procedure under local anesthesia per patient. Treatment decisions were not altered by the results of the SNP under LA. Although we analyzed only twelve patients, we are convinced that the primary aim of our study (that is reducing the number of two-stage surgical procedures under general anesthesia with 30\%) would not have been achieved by finishing the study.

The median number of sentinel nodes removed in our study was similar between the two groups. Two previous cohort studies on SNP under LA did not show a difference in number of sentinel nodes removed as well $[5,7]$. Median duration of SNP under LA was significantly longer than under general anesthesia $(p=0.01)$ in our study. Although duration of SNP under LA was investigated by several study groups, it has never been studied in a randomized controlled trial. Median duration of SNP under LA in our study (median 26 minutes, IQR 7.5 minutes) was comparable to duration of SNP under LA in previous studies (mean durations ranging from 16 to 45 minutes) [5-8]. In our study, patients experienced too much pain during SNP under LA (3 of 5 patients scored 4 or more on NPRS). Both the longer duration of SNP under LA and pain experienced during the procedure are arguments that can be applied against SNP under LA.

One previous study on SNP under LA evaluated psychological impact of the procedure. They stated that " $100 \%$ of patients expressed the belief that the procedure under LA made communication with the physician in the charge of the therapy easier" [21]. However, no validated psychometric questionnaires on QoL, anxiety and depressive symptoms were used. In our study, state anxiety scores were highest at baseline and diminished significantly at all follow-up moments when analyzing all patients collectively. Mean baseline state 
Leenders et al., Breast Cancer Reports 2020,

anxiety score was 45.0 , which is much higher than the mean score of 34 to 36 in a healthy population [14]. The baseline questionnaires were completed at randomization, which is within one week after breast cancer diagnosis. Therefore, the most obvious explanation for the temporary elevation of state anxiety is the breast cancer diagnosis itself. This higher prevalence of anxiety at breast cancer diagnosis is consistent with previous studies [13]. The physical health domain scores of the WHOQOL-bref were significantly lower during followup compared with baseline in the SNP under LA group. This was not observed in the usual care group. Thus, SNP under LA seems to have a negative impact on physical health. This might be explained by the fact that an extra procedure is performed resulting in increased awareness of physical discomfort. Further, the SNP under LA causes additional moments of pain (both during and after the procedure) that might negatively influence physical health. These adverse effects on physical health persist for at least six months.

To our knowledge, this is the only randomized clinical trial investigating the role of SNP under LA in breast cancer patients. Although the aim of the study was well funded and the study was well designed, the timing of the study was unfortunate, since the recent landmark publications of the ACOSOG Z0011, IBCSG 23-01 and AATRM 048/13/2000 studies [2-4] rapidly changed daily practice. Consequently, the study was prematurely closed after inclusion of only twelve patients. Analysis of the end points of these twelve patients resulted in some valuable lessons; SNP under LA does not reduce the number of two-stage surgical procedures, operating time under local anesthesia is longer, too much pain is experienced during the procedure and it results in lower QoL for at least six months. Performing SNP under LA is therefore discouraged. However, if SNP under LA is indicated, it is a safe procedure that does not harm long-term locoregional control [5-9].

\section{Competing interests}

The authors declare that they have no competing interests.

\section{Authors' contributions}

\begin{tabular}{|l|c|c|c|c|c|}
\hline Authors' contributions & ML & SG & ALC & RM & HS \\
\hline Research concept and design & $\checkmark$ & $\checkmark$ & $\checkmark$ & $\checkmark$ & $\checkmark$ \\
\hline Collection and/or assembly of data & $\checkmark$ & $\checkmark$ & $\checkmark$ & $\checkmark$ & $\checkmark$ \\
\hline Data analysis and interpretation & $\checkmark$ & -- & -- & -- & $\checkmark$ \\
\hline Writing the article & $\checkmark$ & -- & -- & -- & $\checkmark$ \\
\hline Critical revision of the article & -- & $\checkmark$ & $\checkmark$ & $\checkmark$ & $\checkmark$ \\
\hline Final approval of article & $\checkmark$ & $\checkmark$ & $\checkmark$ & $\checkmark$ & $\checkmark$ \\
\hline Statistical analysis & $\checkmark$ & -- & -- & -- & $\checkmark$ \\
\hline
\end{tabular}

\section{Acknowledgement}

This work was supported by Foreest Medical School (grant number 394). This funding source had no role in the design of this study, nor in its execution, analyses or interpretation of data.

Publication history

Editor: Wei-Hsiung Yang, Mercer University School of Medicine, USA. Received: 05-Aug-2020 Final Revised: 12-Sept-2020

Accepted: 26-Sept-2020 Published: 11-Oct-2020

\section{References}

1. Mainiero MB. Regional Lymph Node Staging in Breast Cancer: the Increasing Role of Imaging and Ultrasound-Guided Axillary Lymph Node Fine Needle Aspiration. Radiol Clin North Am 2010; 48(5): 989-997.

2. Giuliano AE, Hunt KK, Ballman KV, Beitsch PD, Whitworth PW, Blumencranz PW et al. Axillary

3. dissection vs no axillary dissection in women with invasive breast cancer and sentinel node metastasis. A randomized clinical trial. JAMA 2011; 305(6): 569-575.

4. Galimberti V, Cole BF, Zurrida S, Viale G, Luini A, Veronesi P et al. Axillary dissection versus no axillary dissection in patients with sentinel-node micrometastases (IBCSG 23-01): a phase 3 randomised controlled trial. Lancet Oncol 2013; 14: 297e305.

5. Solá M, Alberoo JA, Fraile M, Santesteban P, Ramos M, Fabregas $R$ et al. Complete axillary lymph node dissection versus clinical follow-up in breast cancer patients with sentinel node micrometastasis: final results from the multicentre clinical trial AATRM 048/13/2000. Ann Surg Oncol 2013; 20: 120e127.

6. Smidt ML, Janssen CM, Barendregt WB, Wobbes T, Strobbe LJ. Sentinel lymph node biopsy performed under local anaesthesia is feasible. Am J Surg 2004; 187(6): 684-687.

7. Luini A, Caldarella P, Gatti G, Veronesi P, Vento AR, Naninato P et al. The sentinel node biopsy under local anaesthesia in breast cancer: advantages and problems, how the technique influenced the activity of a breast surgery department; update from the European Institute of Oncology with more than 1000 cases. Breast 2007; 16(5): 527-532.

8. Kongdan Y, Chirappapha P, Lertsithichai P. Effectiveness and reliability of sentinel lymph node biopsy under local anaesthesia for breast cancer. Breast 2008; 17(5): 528-531.

9. Gauthier T, Mollard J, Fermeaux V, Kapella M, Aubard Y. Axillary recurrence after negative sentinel lymph node biopsy under local anaesthesia in breast cancer. Eur J Surg Oncol 2009; 35(5): 464-468.

10. Groetelaers RPTGC, van Berlo CL, Nijhuis PH, Schapers RF, Gerritsen HA. Axillary recurrences after negative sentinel lymph node biopsy under local anaesthesia for breast cancer: a follow-up study after 5 years. Eur J Surg Oncol 2009; 35(2): 159-163.

11. Leenders MWH, Richir MC, Broeders M, Moormann GHRM, Mollema $\mathrm{R}$, Lopes Cardozo AMF et al. Axillary staging by ultrasound-guided fine needle aspiration cytology in breast cancer patients. Still up to date? The Breast Journal 2013; 19(6):637-642.

12. Fann JR, Thomas-Rich AM, Katon WJ, Cowley D, Pepping M, McGregor BA et al. Major depression after breast cancer: a review of epidemiology and treatment, General Hospital Psychiatry 2008;30:112-126.

13. Christensen S, Zachariae R, Bonde Jensen A, Vaeth M, Moller S, Ravnsbaek J et al. Prevalence and risk of depressive symptoms 3-4 months postsurgery in a Burgess C, Cornelius V, Love S, Graham J, Richards M, Ramirez A. Depression and anxiety in women with early breast cancer: five year observational cohort study, BMJ 2005;330:702.

14. Spielberger CD, Gorsuch RL, Lushene R, Vagg PR, Jacobs GA. Manual for the State-Trait Anxiety Inventory 1983. Palo Alto, CA: Consulting Psychologists Press.

15. Van der Bij AK, de Weerd C, Cikot RJ, Steegers EA, Braspenning JC. Validation of the dutch short form of the state scale of the Spielberger State-Trait Anxiety Inventory: considerations for usage in screening outcomes. Community Genet 2003;6:84-87.

16. Skevington SM, Lofty M, O'Connell KA. The World Health Organization's WHOQOL-BREF quality of life assessment: Psychometric properties and results of the international field trial. A report from the WHOQOL Group. Quality of Life Research 2004; 13: 299-310.

17. Hann D, Winter K, Jacobsen P. Measurement of depressive symptoms in cancer patients: evaluation of the Centre for Epidemiological Studies Depression Scale (CES-D). J Psychosom Res 1999; 46: 437-443.

18. Schroevers MJ, Sanderman R, van Sonderen E, Ranchor AV. The evaluation of the Centre for Epidemiological Studies Depression (CES-D) scale: Depressed and Positive affect in cancer patients and healthy reference subjects. Qual Life Res 2000; 9: 1015-1029. 
Leenders et al., Breast Cancer Reports 2020,

http://www.hoajonline.com/journals/pdf/2057-1631-7-3.pdf

19. Bouma J, Ranchor AV, Sanderman R, Sonderen van E. Het meten van symptomen van depressie met de CES-D. Een handleiding. 1995.

Noordelijk Centrum voor Gezondheidsvraagstukken. Rijksuniversiteit Groningen.

20. Lambert K, Mokbel K. Does post-mastectomy radiotherapy represent a contraindication to skin-sparing mastectomy and immediate reconstruction: An update.Surgical Oncology 2012; 21: e67-e74.

21. Luini A, Gatti G, Zurrida S, Galimberti V, Paganelli G, Naninato P et al. The sentinel lymph node biopsy under local anaesthesia in breast carcinoma: experience of the European Institute of Oncology and impact on quality of life. Breast Cancer Research and Treatment 2005; 89: 69-74.

\section{Citation:}

Leenders M, Go S, Cardozo AL, Mollema R and Schreurs H. Sentinel node procedure under local anesthesia in breast cancer patients. Lessons learned from a prematurely closed randomized clinical trial. Breast Cancer Rep. 2020; 7:3.

http://dx.doi.org/10.7243/2057-1631-7-3 